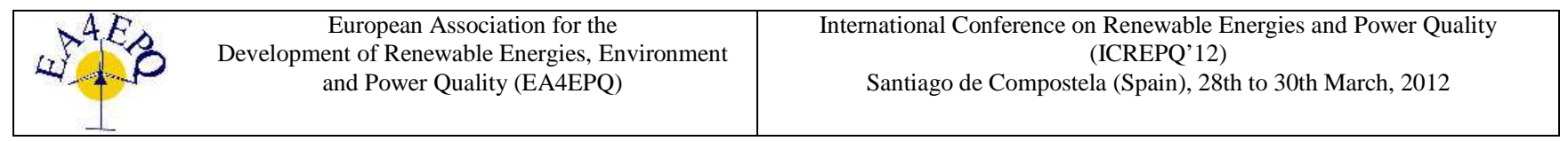

\title{
Inertia and Governor Ramp Rate Constrained Economic Dispatch to Assess Primary Frequency Response Adequacy
}

\author{
Hector Chavez ${ }^{1}$, and Ross Baldick ${ }^{2}$ \\ ${ }^{1,2}$ Department of Electrical Engineering \\ University of Texas at Austin \\ 1 University Station C0803 Austin, TX 78712-0240 U.S. \\ Phone/Fax number:+0034 986 812685, e-mail: h.chavez.orostica@gmail.com, baldick@ece.utexas.edu
}

\begin{abstract}
The contribution of non-dispatchable, renewable generation to both total grid inertia and grid frequency response is significantly lesser than that of dispatchable, thermal generation. In large non-dispatchable generation penetration scenarios, the effect of inertia and frequency response diminution on primary frequency control reserves adequacy will be important.

This paper develops a constraint on the economic dispatch problem that assures that frequency nadirs after a given sudden loss of generations will not be lower than a given limit. This constraint considers system inertia and governor ramp rate limits. A simulation using the IEEE Reliability Test System is presented.
\end{abstract}

\section{Key words}

Primary Frequency Response Adequacy, Economic Dispatch, Non-dispatchable generation, Renewable Energy Allocation.

\section{Introduction}

The increase in renewable electricity sources in North American interconnected systems is associated with deterioration in the primary frequency response of those systems [1]. This is not a main concern of system operators, since primary frequency control reserves (PFCR) have been adequate. However, as more non-dispatchable, renewable generation is added to the grid, the capability of the system to respond to sudden losses of generation (contingencies) will reduce, because

i) units with governor providing primary frequency response are replaced by non-dispatchable units that present low to zero contribution to total system frequency response, and

ii) units synchronously connected providing inertial response are replaced by non-dispatchable, asynchronous units that present low to zero contribution to the total inertia of the system.

The points described above suggest that both system inertia and governor response capability are elements that should be included in an economic dispatch (ED) formulation to assure frequency reliability. Current assessments on PFCR adequacy are either dynamically insufficient or not suitable for an ED implementation.

The phenomena described in (i) and (ii) above are reported in [3]. This report explores the accommodation of synthetic inertia and primary frequency response from asynchronous units (wind turbines, HVDC lines) to withstand i) and ii) in the English system. The report assesses PFCR adequacy by performing a trial and error simulation process to define the appropriate amounts of PFCR and inertia (from both synchronous and asynchronous sources) so that system frequency nadirs (point C from Fig. 1) satisfy the English system requirement. For low load and high wind scenarios, PFCR procurement was not adequate for any synchronous (dispatchable) governor configuration, and faster governor response units (from asynchronous generation) were needed to satisfy the nadir-based compliance. One important observation made on wind turbines providing frequency response is that such control algorithms need to curtail wind output, which represents a cost that needs to be considered. This work recommends that "proposals should be developed for the appropriate obligations and/or market arrangements to ensure sufficient frequency response capability is available to maintain system security for anticipated future generation and demand patterns."

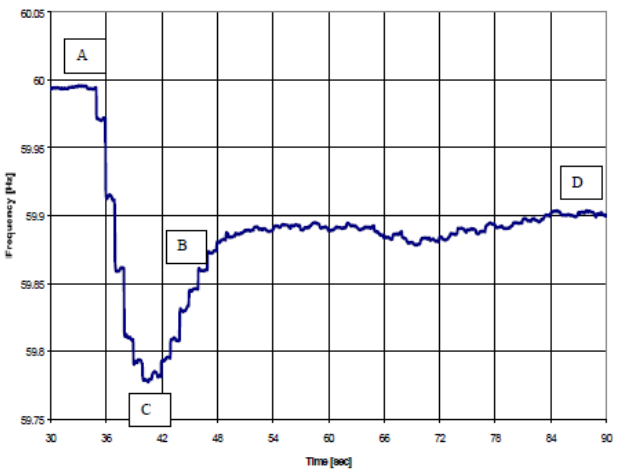

Fig. 1. NERC definition of frequency excursion [2].

\footnotetext{
${ }^{1}$ This research was supported in part by both the National Science Foundation under award ECCS-1065224 and the Fulbright Foundation.
} 
A simulation of two North American interconnections with wind energy penetration levels of $3 \%$ and $13 \%$ for WECC and ERCOT respectively and considering the worst governor ramping capability conditions of such systems is presented in [1]. This report defines adequacy metrics considering empirical data of frequency nadirs (point $\mathrm{C}$ in Fig. 1) and system governor ramping capability. Since these metrics are based on empirical contingency measurements, they do not provide an assessment to be included in an ED formulation depending on structural parameters of the grid.

The work presented in [4] develops a constraint on PFCR in a unit commitment (UC) formulation. This paper assumes that all generators respond proportional to its droop characteristics, and that governor response is limited to committed PFCR. This approach assures that the governor steady state frequency response (points B and $\mathrm{D}$ in Fig. 1) will be adequate, but neglects the transient nadir (point $C$ ) that depends on the inertia of the system and the ramping capability of unit governors, as described in [1], and [3].

System operators in North America establish PFCR obligations in ED and UC without considering system inertia or governor ramping capability [1]. IES and WECC required that total PFCR (MW) be a percentage of total load, and ERCOT considers the two largest generators in the system to define total PFCR (MW) obligation. In addition to this, the capacity margin of these systems is always larger than PFCR obligation, so that there is always more governor responsive capacity than needed (some generator do not have governor in service; ERCOT required that all generators have governor in service except wind and nuclear-powered ones). However, these requirements are not sufficient for all possible inertia and ramping capability conditions in a high non-dispatchable generation scenario.

This papers first formulates a condition on the overall governor response of the system that assures that system frequency will not decay bellow certain pre-defined value, given an inertia condition and a contingency magnitude. This overall condition on system governor capacity is formulated as a condition on each unit governor characteristics, and a simulation is presented to observe the results of implementing such a constraint. Then, a generalization of the constraint is presented so that it is suitable to be implemented in a UC formulation.

\section{Primary Frequency Control Description}

The role of primary frequency control is to prevent frequency from extreme deviations after a contingency. Primary frequency control is provided by

system inertia of rotational masses that store kinetic energy,

a governor that automatically varies the power set point of a generator in response to a frequency deviation, and

interruptible loads that are automatically disconnected from the grid by under frequency relays.

\section{A. Inertia}

A rotational mass stores kinetic energy proportional to its mass and the square of its angular velocity. If system frequency drops, the change in kinetic energy is liberated to the grid, and tends to arrest the frequency drop (if frequency increases, energy is withdrawn). Inertia is the first instance of system frequency response.

\section{B. Governor}

A governor is a local feedback controller that senses the angular velocity of generator shaft and acts on the steam valve of generator turbine. The gain of the feedback loop is normally call the droop characteristic of the governor, and relates the fraction of capacity that the governor deploys in response to a given frequency deviation. Also, governors have a frequency dead band. A detailed description of governors can be seen in [5].

\section{Responsive Loads}

A load can be committed to automatically disconnect from the grid in response to frequency deviations. This is different from under frequency relays, because the latter is a forced, undesirable condition that system operators must avoid, and can cause instability and rolling blackouts. Responsive loads are committed to act in case of a contingency, and its activation does (must) not cause instability. A good example of a responsive load definition is ERCOT's LaaRs [6].

\section{Primary Frequency Control Adequacy}

The assumptions of this paper regarding system frequency dynamics are:

I) during the first 2-20 second after a contingency, the frequency response of the system is only given by the elements described in section 2 above. Secondary frequency control normally responds 1 minute after a contingency [2],

II) the inertia of the system is given by the sum of the inertias of all generators online within the interconnection. This is consistent with the mechanical definition of system inertia [7],

III) the overall system governor response can be modeled as a constant ramp rate, a dead time, and an output limit.

\section{A. PFCR adequacy criterion}

This paper defines PFCR adequacy as the capability of PFCR resources to prevent frequency from decaying bellow a given limit after a contingency of magnitude less than or equal to a given value, where

$f_{M I N} \quad$ : minimum frequency allowed $(\mathrm{Hz})$,

$\Delta f_{M I N} \quad$ : frequency deviation allowed $(\mathrm{Hz})$, and

$\Delta P_{M A X} \quad$ : maximum contingency magnitude (MW).

\section{B. Dynamic Conditions to Satisfying the Adequacy Criterion}

A general description of frequency dynamics considering assumptions a) and b) above can be derived from the swing equation [7]:

$$
\frac{d \Delta f(t)}{d t}=\frac{1}{T_{H}}\left(P_{g}(t)-\Delta P\right)
$$


where

$\Delta f(t) \quad$ : frequency deviation $(\mathrm{Hz})$

$T_{H} \quad$ : inertia coefficient $\left(\mathrm{MW} \cdot \mathrm{s} \cdot \mathrm{Hz}^{-1}\right)$

$P_{g}(t) \quad$ : governor response (MW)

$\Delta P \quad$ : contingency magnitude (MW)

After a contingency, $P_{g}(\mathrm{t})$ increases at a maximum ramp rate as considered in [8],[9]. This maximum ramp rate can be approximated by a constant value $R$, as stated in point III above. This approximation is shown in Fig. 2.

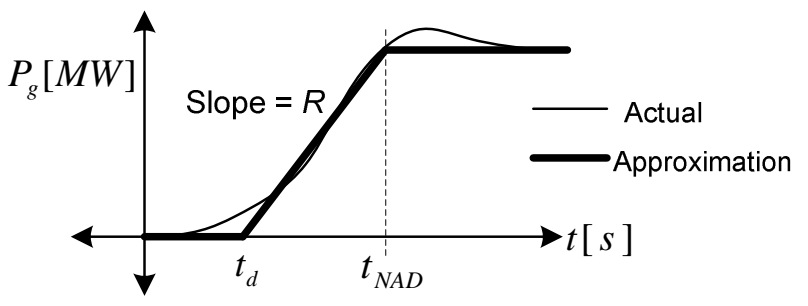

Fig. 2. Governor response approximation

The approximation assumes that there is a dead time $t_{d}$ that is a common feature of governor response [5]. A condition for the nadir time $t_{N A D}$ considering the simplification in Fig. 2 and (1) is given by

$$
\begin{aligned}
\frac{d \Delta f\left(t_{N A D}\right)}{d t}=0 & \Rightarrow R\left(t_{N A D}-t_{d}\right)=\Delta P \\
& \Rightarrow t_{N A D}=\frac{\Delta P}{R}+t_{d}
\end{aligned}
$$

This considers that frequency derivative must be cero at the nadir. Replacing (2) in (1), the value of the nadir is given by:

$$
\begin{aligned}
\Delta f_{N A D}-\Delta f(0) & =\frac{1}{T_{H}}\left(\int_{0}^{t_{d}}(-\Delta P) d t+\int_{t_{d}}^{t_{N A D}}(R t-\Delta P) d t\right) \\
& =\frac{-1}{T_{H}}\left(\frac{\Delta P^{2}}{2 R}+\Delta P t_{d}\right)
\end{aligned}
$$

The adequacy criterion described in $A$ above is satisfied if $f_{M I N} \leq f_{N A D}$ as shown in Fig. 3 .

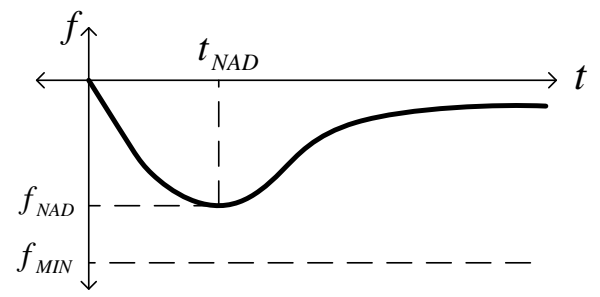

Fig. 3. Adequacy criterion

Then, considering a pre-contingency frequency deviation $\Delta f(0)$ approximately zero, a sufficient condition that assures the adequacy criterion is given by:

$$
\Delta f_{M I N} \leq \Delta f_{N A D} \Rightarrow R \geq \frac{\Delta P^{2}}{2\left(T_{H} \Delta f_{M I N}-\Delta P t_{d}\right)}
$$

This constraint assures that point $\mathrm{C}$ in Fig. 1 will be higher than $\Delta f_{M I N}$ if a contingency of magnitude less than or equal to $\Delta P$ happens. However, system operators have under frequency relays that operate after couple of second of sustained deviation, so an analysis on steady state governor response is needed. This paper does not include such an analysis, but [4] has a good description of it.

\section{Individual Governor Constraint}

Expression (4) defines a constraint on an approximate, linear overall ramping governor response that satisfies the adequacy criterion. However, economic dispatch formulations require an expression that involves individual unit characteristics.

\section{A. Relationship between $R$ and Individual Unit Ramp Rates}

The constraint (4) considers an overall ramp rate $R$, but a relationship between this value and individual ramp rates is needed for an ED formulation. For such an analysis consider

$i \in I \quad$ : set of generators,

$p_{i} \quad$ : generator $i$ committed cap. for energy (MW),

$y_{i} \quad:$ generator $i$ committed cap. for PFCR (MW),

$r_{i} \quad$ : generator $i$ governor ramp rate $(\mathrm{MW} / \mathrm{s})$,

$h_{i} \quad:$ generator $i$ inertia coefficient $\left(\mathrm{MW} \cdot \mathrm{s} \cdot \mathrm{Hz}^{-1}\right)$,

$p_{i}{ }^{-} \quad$ : generator $i$ maximum output (MW),

$p_{i,} \quad \quad$ : generator $i$ minimum output (MW), and

$y_{i}{ }^{-} \quad$ : generator $i$ maximum PFCR (MW).

Although in general, $p_{i},{ }^{-}-p_{i} \neq y_{i}$ (a generator can commit to provide other ancillary services, such as secondary frequency reserves), $y_{i}$ will be considered as the governor response output limit.

Sufficient conditions on PFCR that assures adequacy as described above can be derived from $\Delta P, y_{i}$, and $t_{N A D}$. Condition (4) requires that the system deploy $\triangle P(\mathrm{MW})$ at a constant ramp rate $R$, which is equivalent to deploy $\triangle P(\mathrm{MW})$ in $t_{N A D}$ seconds at a constant ramp rate. Then, individual governor deployments must sum up to $\Delta P$ and be deployed in $t_{N A D}$ seconds or before, it is to say

$$
\begin{aligned}
& \sum_{i \in J} y_{j} \geq \Delta P \\
& y_{i} \leq r_{i} t_{N A D}, \quad i \in I \\
& y_{i} \leq \bar{y}_{i}, \quad i \in I
\end{aligned}
$$

where $J$ is the set of generators except the largest. A generator can offer an amount of capacity that is not fully deployable in $t_{N A D}$ seconds; condition (6) prevents this from happening, so (5) only includes capacity that is fully deployable in $t_{N A D}$ seconds. This is a sufficient condition for $f_{M I N} \leq f_{N A D}$.

Condition (6) can be rewritten using (2) and (4):

$$
y_{i} \leq r_{i} \frac{\Delta P}{R}=2 r_{i}\left(\frac{T_{H} \Delta f_{M I N}-\Delta P t_{c}}{\Delta P}\right), \quad i \in I
$$

where the inertia coefficient $T_{H}$ is the sum of all inertia 
coefficients of on line generator within the grid

$$
T_{H}=\sum_{i \in I} h_{i}
$$

$h_{i}$ can be computed from the traditional definition of inertia [7] as follows:

$$
h_{i}=\frac{2 P_{B A S E} H_{i}}{60}, \quad i \in I
$$

where $P_{B A S E}(\mathrm{MW})$ is the machine power base, and $H_{i}$ is the traditional inertia parameter.

\section{B. Constraint Accuracy}

The dead time is a combination of individual unit dead times, so the overall dead time is hard to compute theoretically [9]. This paper will consider an estimate based on simulated governor responses.

Although the linear approximation described in Fig. 2 is in general a good description of governor response, there is always a difference with the actual response. This difference must be always considered so that the approximation is slower than the actual system (as shown in Fig. 2) and $f_{M I N} \leq f_{N A D}$ holds.

One important accuracy factor is generators that deploy all their PFCR before $t_{N A D}$. From (5) and (6), the overall ramp rate $R$ is given by the sum of individual ramp rates. If a generator deploys all its capacity before $t_{N A D}$, (6) will not be binding for that generator, and the equivalent ramp rate contribution to $R$ will be smaller than the actual ramp rate of that generator. In such a case, the actual response will be faster than estimated, and the difference between $f_{M I N}$ and $f_{N A D}$ will be large. Thus, a tighter $f_{M I N}$ will lead to a smaller $f_{M I N}-f_{N A D}$ difference.

\section{Economic Dispatch Formulation}

An ED formulation including both energy and PFCR procurement for one period is considered. This one-period formulation will consider that commitment decisions are known, so that total inertia $T_{H}$ is constant and known.

The system must meet a demand $D(\mathrm{MW})$ during a single period. Generator's offers are considered to be convex [10]:

$e_{i}\left(p_{i}\right) \quad$ : generator $i$ energy offer $(\$ / \mathrm{h})$

$s_{i}\left(y_{i}\right) \quad$ : generator $i$ PFCR offer (\$/MW)

Then, the inertia and ramp rate constrained ED can be formulated as follows:

$$
\begin{aligned}
& \min \sum_{i \in I}\left(e_{i}\left(p_{i}\right)+s_{i}\left(y_{i}\right)\right) \\
& \text { s.t. } \\
& \sum_{i \in I} p_{i}=D \\
& \sum_{i \in J} y_{j} \geq \Delta P_{M A X} \\
& y_{i} \leq 2 r_{i}\left(\frac{T_{H} \Delta f_{M I N}-\Delta P t_{c}}{\Delta P}\right), \quad i \in I
\end{aligned}
$$

$$
\begin{aligned}
& 0 \leq y_{i} \leq \bar{y}_{i}, \quad i \in I \\
& p_{i} \geq \underline{p}_{i}, \quad i \in I \\
& p_{i}+y_{i} \leq \bar{p}_{i}, \quad i \in I
\end{aligned}
$$

The system (10) can be cast in a more convenient matrix formulation. Let $n=|I|$. Let $x \in \mathfrak{R}^{2 n}$ be the vector that concatenates both $p_{i}$ and $y_{i}, i \in I, A \in \mathfrak{R}^{2 \mathrm{n}}, b \in \mathfrak{R}, C$ $\in \mathfrak{R}^{(10 n+1) \times 2 n}, d \in \mathfrak{R}^{10 n+1}$, and $f(x): \mathfrak{R}^{2 n} \rightarrow \mathfrak{R}$ such that

$$
\begin{aligned}
& A=\left[\begin{array}{ll}
1_{1 \times n} & 0_{1 \times n}
\end{array}\right], b=D, \\
& C=\left[\begin{array}{cc}
0_{1 \times n} & -1_{1 \times n} \\
0_{n \times n} & I_{n \times n} \\
0_{n \times n} & I_{n \times n} \\
0_{n \times n} & -I_{n \times n} \\
-I_{n \times n} & 0_{n \times n} \\
I_{n \times n} & I_{n \times n}
\end{array}\right], \quad d=\left[\begin{array}{c}
-\Delta P_{M A X} \\
\alpha r \\
\bar{y} \\
0 \\
-\underline{p} \\
\bar{p}
\end{array}\right], \\
& f(x)=\sum_{i \in I}\left(e_{i}\left(p_{i}\right)+s_{i}\left(y_{i}\right)\right)
\end{aligned}
$$

where

$I_{n \times n} \quad: n \times n$ identity matrix,

$1_{1 \times n}: 1 \times n$ all ones row vector,

$0_{1 \times n}: 1 \times n$ zero row vector

$0_{\mathrm{n} \times n}: n \times n$ zero matrix

$p,^{-} \quad$ : generator maxima output vector,

$p$, $_{-} \quad$ : generator minima output vector,

$y,^{-} \quad$ : capacity for PFCR upper bound vector,

$r \quad:$ ramp rates vector, and

$$
\alpha=2 \frac{T_{H} \Delta f_{M I N}-\Delta P t_{c}}{\Delta P}
$$

With these definitions, problem (10) can be formulated as follows

$$
\min (f(x) \mid A x=b, C x \leq d)
$$

Since $f$ is a convex function, the first order optimality conditions [10] are sufficient to solve problem (12).

\section{One Area IEEE RTS 96 Example}

The IEEE reliability test system [11] is used to implement the inertia and ramp rate constrained ED presented above. PowerWorld is used to ran the simulations (Matlab was used to minimize de ED). The largest generators in the system are two nuclear-powered 400-MW units, so a trip of one of these units is considered.

Assuming that most dispatchable generators have governor, the effect of transmission constraint on governor deployments is negligible. After a contingency, the overall frequency response is provided by small contributions from all generator, and line overloads, if any, will also be small. Then, transmission is not included in the ED formulation.

Generator offers are associated to the quadratic 
equation $\operatorname{Pr}(Q)=a+b Q+c Q^{2}(\$ / \mathrm{h})$, where $\operatorname{Pr}(Q)$ is the price at which the generator offer a power $Q$ per hour (offers for PFCR are considered zero). The prices of fuel are as follows [12]: 22.81\$/MMBTU (\#2 oil), 18.13\$/MMBTU (\#6 oil), 2.41\$/MMBTU (coal), and 2\$/MMBTU (nuclear) (Hydro is assumed as indicated in table I). Minimum generator outputs are not specified in [11], so zero is assumed as minimum outputs $p$, . Maximum PFCR level $y,{ }^{-}$is assumed $30 \%$ of total capacity. The dead time $t_{c}$ was set to $0.5 \mathrm{~s}$ by observation of simulated governor responses. Demand is $D=3999.4$ MW, as presented in [11].

Governor models are considered as recommended in [13], and a detailed description of these models is shown in [14]. Oil units are associated to GGOV1 governor, coal units to IEEEG1, and hydro units to HYGOV4. Nuclear units are considered non-responsive (they are always fully dispatch by economic merit). Governor ramp rates are directly measured from simulations of such models and the dynamic parameters presented in [11].

Table I shows simulation parameters. Total inertia does not consider the inertia of the unit that trips, so table II describing inertia parameters only considers one 400-MW nuclear unit.

Table I. - Generator Parameters.

\begin{tabular}{|c|c|c|c|c|c|c|c|}
\hline \multirow{2}{*}{ Unit } & \multirow{2}{*}{$\begin{array}{c}\text { \# of } \\
\text { units }\end{array}$} & \multirow{2}{*}{$\begin{array}{c}\text { Gov. } \\
\text { Model }\end{array}$} & \multirow{2}{*}{$\begin{array}{c}\boldsymbol{r}_{\boldsymbol{i}} \\
(\mathrm{MW} / \mathbf{s})\end{array}$} & \multirow{2}{*}{$\begin{array}{l}p_{i}{ }^{-} \\
\text {(MW) }\end{array}$} & \multicolumn{3}{|c|}{ Generator offer } \\
\hline & & & & & $a$ & $b$ & $c$ \\
\hline U12 & 5 & GGOV1 & 1.13 & 12 & 305 & 157 & 2.87 \\
\hline U20 & 4 & GGOV1 & 1.9 & 20 & 301 & 166 & 2.1 \\
\hline U50 & 6 & HYGOV4 & 1 & 50 & 200 & 11 & 0.01 \\
\hline U76 & 4 & IEEEG1 & 7.6 & 76 & 323 & 19 & 0.07 \\
\hline U100 & 3 & GGOV1 & 9.3 & 100 & 2352 & 136 & 0.22 \\
\hline U155 & 4 & IEEEG1 & 15.6 & 155 & 444 & 18.2 & 0.01 \\
\hline U197 & 3 & GGOV1 & 18 & 197 & 698 & 139 & 0.09 \\
\hline U350 & 1 & IEEEG1 & 34.4 & 350 & 780 & 17.9 & 0.01 \\
\hline U400 & 2 & --- & --- & 400 & 410 & 6.2 & 0 \\
\hline
\end{tabular}

Table II. - Inertia parameters

\begin{tabular}{|c|c|c|c|c|}
\hline Unit & $\begin{array}{l}\text { \# of } \\
\text { units }\end{array}$ & $\begin{array}{c}\text { MVA } \\
\text { Base }\end{array}$ & $\begin{array}{c}H \\
(\mathrm{MJ} / \mathrm{MW})\end{array}$ & $\begin{array}{l}T_{H} \text { (all units) } \\
\left(\mathrm{MW} \cdot \mathrm{s} \cdot \mathrm{Hz}^{-1}\right)\end{array}$ \\
\hline U12 & 5 & 14 & 2.8 & 3.26666667 \\
\hline $\mathrm{U} 20$ & 4 & 24 & 2.8 & 4.48 \\
\hline U50 & 6 & 53 & 3.5 & 18.55 \\
\hline U76 & 4 & 89 & 3 & 17.8 \\
\hline U100 & 3 & 118 & 2.8 & 16.52 \\
\hline U155 & 4 & 182 & 3 & 36.4 \\
\hline U197 & 3 & 232 & 2.8 & 32.48 \\
\hline U350 & 1 & 412 & 3 & 20.6 \\
\hline U400 & 1 & 471 & 5 & 39.25 \\
\hline \multicolumn{4}{|c|}{ Total $T_{H}$} & 189.34667 \\
\hline
\end{tabular}

A simulation of different nadir requirements is presented in table III, table IV, table V, and Fig. 4.

Table III. - Dispatch costs and prices

\begin{tabular}{|l|r|r|r|}
\hline $\boldsymbol{f}_{\text {MIN }}(\mathbf{H z})$ & $\mathbf{0}$ & $\mathbf{5 7}$ & $\mathbf{5 8 . 5}$ \\
\hline Energy Price $(\$ / \mathrm{MWh})$ & 160.48 & 167.53 & 176.62 \\
\hline Energy Payment $(\$)$ & $641,823.7$ & $670,019.4$ & 706,374 \\
\hline Reserve Price $(\$)$ & 0.001 & 143.01 & 170.41 \\
\hline Reserve Payment $(\$)$ & 0.4 & 57,204 & 68,164 \\
\hline Total $(\$)$ & $641,824.1$ & $727,223.4$ & 774,538 \\
\hline
\end{tabular}

Table IV. - Dispatch quantities

\begin{tabular}{|l|r|r|r|r|r|r|}
\hline \multirow{3}{*}{ Unit } & \multicolumn{3}{|c|}{ Energy (MW-h) } & \multicolumn{3}{c|}{ PFCR (MW) } \\
\cline { 2 - 7 } & $\begin{array}{c}\boldsymbol{f}_{\text {MIN }}= \\
\mathbf{0 ~ H z}\end{array}$ & $\begin{array}{c}\boldsymbol{f}_{\text {MIN }}= \\
\mathbf{5 7 ~ H z}\end{array}$ & $\begin{array}{c}\boldsymbol{f}_{\text {MIN }}= \\
\mathbf{5 8 . 5} \mathbf{H z}\end{array}$ & $\begin{array}{c}\boldsymbol{f}_{\text {MIN }}= \\
\mathbf{0 ~ H z}\end{array}$ & $\begin{array}{c}\boldsymbol{f}_{\text {MIN }}= \\
\mathbf{5 7 ~ H z}\end{array}$ & $\begin{array}{c}\boldsymbol{f}_{\text {MIN }}= \\
\mathbf{5 8 . 5 H z}\end{array}$ \\
\hline U12 & 0.6 & 1.8 & 3.41 & 6.5 & 3.4 & 1.7 \\
\hline U20 & 17.5 & 14.2 & 17.08 & 2.5 & 5.8 & 2.9 \\
\hline U50 & 50 & 50 & 43.57 & 0 & 0 & 6.42 \\
\hline U76 & 76 & 54.4 & 65.2 & 0 & 21.6 & 10.8 \\
\hline U100 & 55.15 & 71.18 & 85.68 & 44.75 & 28.82 & 14.32 \\
\hline U155 & 155 & 155 & 132.92 & 0 & 0 & 22.07 \\
\hline U197 & 119.4 & 140.71 & 168.85 & 69.12 & 56.29 & 28.14 \\
\hline U350 & 350 & 331.35 & 300.02 & 0 & 18.65 & 49.98 \\
\hline U400 & 400 & 400 & 367.52 & 0 & 0 & 32.47 \\
\hline
\end{tabular}

Table V. - Frequency Response Results

\begin{tabular}{|l|r|r|r|}
\hline$f_{\text {MIN }}(\mathbf{H z})$ & $\mathbf{0}$ & $\mathbf{5 7}$ & $\mathbf{5 8 . 5}$ \\
\hline$f_{\text {NAD }}(\mathrm{Hz})$ & 52.832 & 57.774 & 58.536 \\
\hline$\alpha(\mathrm{s})$ & --- & 3.8402 & 2.4201 \\
\hline
\end{tabular}
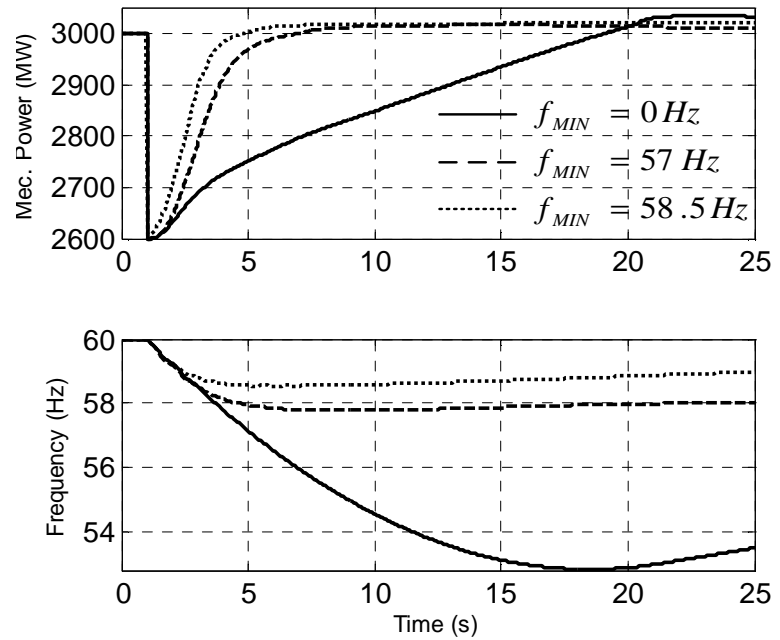

Fig. 5. Frequency response. Mechanical power (a), and frequency (b).

The results in table III and Fig. 3 are consistent with the definition of the constraint. The unconstrained case $\left(f_{M I N}=0\right)$ does not consider $(6)$, so PFCR are only defined by (5). The difference between $f_{M I N}$ and $f_{N A D}$ is smaller for a tighter $f_{M I N}$, as described in section $4, B$.

Dispatch results show a more expensive constrained ED. The constraint requires that PFCR be shared by more units, so cheap generation is no longer fully dispatched. PFCR prices spike from 0 to about 143 and $170 \$ / \mathrm{MW}$, which represents the opportunity cost of not utilizing cheap capacity to produce energy; that price assures that generators are indifferent between offering capacity in the energy market or as PFCR [15]. This is increased by tighter nadir requirements and larger differences between offers.

\section{UC extension}

Although this paper does not consider a UC formulation [16], (8) can be generalized to be included in a UC formulation. Let $z_{i} \in\{0,1\}, i \in I$, the commitment decision of switching off $\left(z_{i}=0\right)$ or switching on $\left(z_{i}=1\right)$ generator $i$. Then, (9) can be written as

$T_{H}=\sum_{j \in J} h_{j} z_{j}$ 
and $(8)$ in a more general form

$$
y_{i} \leq 2 r_{i}\left(\frac{\Delta f_{M I N} \sum_{i \in J} h_{i} z_{i}-\Delta P t_{c}}{\Delta P}\right), i \in I
$$

This constraint is linear on $y_{i}$ and $z_{i}$, so a solution of the resulting inertia and governor ramp rate constrained UC may present a tractable solution.

\section{Conclusions}

The main effect of the constraint on the dispatch is distributing the PFCR burden among all generators. The unconstrained ED fully dispatches cheap units, so PFCR is carried by large capacity portions from few units. In the constrained case, the same PFCR burden is carried by small contributions of more units. This comes from the fact that the overall ramping capability of the system is the sum of individual generator ramping capabilities, and, as more units provide PFCR, faster is the governor response of the system.

Dispatch costs in inertia and governor ramp rate constrained ED scenarios are higher since there is a need to use more generators to procure PFCR. The new constraint prevents cheap generation from being fully dispatched, and more expensive units set the price.

A UC formulation would give more realistic outcome. The ED formulation presented above considers that commitment decisions are known, so inertia is a constant. In a UC formulation, inertia depends on the commitment decisions as shown in section 8 , so low load conditions will lead to low inertia and governor ramping conditions. Such conditions may be unfeasible in an inertia, governor ramp-rate UC constrained scenario, and the cost of keeping units on line to enforce reliability may exacerbate the increment in dispatch costs with respect to the unconstrained UC optimum. A future work should consider a simulation of such a scenario.

Faster primary frequency response from asynchronous units can reduce costs, as described in [3]. Most commercial wind turbines control schemes have synthetic frequency response algorithms, but they prevent the turbine from producing all its wind power potential. This has a negative effect on reducing $\mathrm{CO}_{2}$ emission, which is the main purpose of using renewable electricity sources. However, the economic benefit from "spilling" wind to improve the frequency response of the system can justify such a policy.

Other fast response, expensive devices can be used to improve the ramping capability of the system without affecting optimal UC dispatch. Fast storage systems can improve the overall ramping capability of the system so that it is no longer necessary to use cheap generation for PFCR to satisfy the adequacy criterion. The saving from improving dispatch costs can represent an economic incentive to using fast storage devices. A future work could analyze both the economic tradeoff and the appropriate market definition to implement such devices as a market product.

This paper does not consider an analysis of steady state governor response (point B in Fig. 1). Also, the contribution of interruptible load needs to be included. A future work should consider an analysis of both steady state governor response and interruptible loads.

\section{References}

[1] J. Eto, J. Undrill, P. Mackin, H. Illian, C. Martinez, M. O’Malley, and K. Coughlin. "Use of Frequency Response Metrics to Assess the Planning and Operating Requirements for Reliable Integration of Variable Renewable Generation". Lawrence Berkeley National Laboratory, Berkeley, CA, Tech. Rep. LBNL- 4142E, Dec. 2010.

[2] NERC Reliability Standards. [Online]. Available: http://www.nerc.com/

[3] NationalGrid Frequency Response Working Group. "Frequency Response Technical Sub-Group Report”. Tech. Rep. Nov. 2011. Available: http://www.nationalgrid.com/NR/rdonlyres/2AFD4C05E169-4636-BF02DC67F80F9C2/50090/FRTSGGroupReport Final.pdf

[4] José F. Restrepo, and Francisco D. Galiana," Unit Commitment With Primary Frequency Regulation Constraints," IEEE Trans. Power Syst., vol. 20, no. 4, Nov. 2005

[5] Dineley, J.L.; Powner, E.T.; , "Power-system governor simulation," Electrical Engineers, Proceedings of the Institution of , vol.111, no.1, pp.115-124, January 1964

[6] ERCOT Methodologies for Determining Ancillary Service Requirements. 2011. [Online]. Available: http://www.ercot.com/

[7] P. Kundur. Power system Stability and Control. New York: McGraw-Hill, 1994, p.584.

[8] P. Ruiz and P. Sauer, "Spinning contingency reserve: Economic value and demand functions," Power Systems, IEEE Transactions on, vol. 23, no. 3, pp. 1071 -1078, Aug. 2008.

[9] Somuah, C.B., Schweppe, F.C. "Economic Dispatch Reserve Allocation," IEEE Trans. Power Apparatus and Syst., vol. PAS100, no. 5, 1981

[10] Baldick, Ross. "Applied optimization: formulation and algorithms for engineering systems."Cambridge, UK ; New York : Cambridge University Press, 2006.

[11] Reliability Test System Task Force, "The IEEE reliability tes system 1996," IEEE Transactions on Power Systems, vol. 14, no. 3, pp. 1010 1020, August 1999.

[12] U.S. Energy Information Administration. "Short $\square$ Term Energy Outlook January 2012”. [Online]. Available: http://38.96.246.204/forecasts/steo/pdf/steo_full.pdf

[13] Les Pereira, John Undrill, Dmitry Kosterev, Donald Davies, and Shawn Patterson. "New Thermal Turbine Governor Modelling for the WECC". $2002 . \quad$ [Online]. Available: http://www.wecc.biz/library/WECC\%20Documents/Documents\%2 0for\%20Generators/New\%20Thermal\%20Governor\%20Modeling/ New\%20Thermal\%20Governor\%20Modeling\%20Report.pdf

[14] PowerWorld Governor Block Diagrams. [Online]. Available: http://www.powerworld.com/Document\%20Library/version.160/B1 ock\%20Diagrams.pdf

[15] Steven Stoft, "Power System Economics: Designing Markets for Electricity", IEEE Press and Wiley Interscience and John Wiley and Sons, Inc., Piscataway, NJ, 2002.

[16] B. Wollenberg and A. Wood, Power Generation, Operation and Control, 2nd ed. New York, NY: John Wiley, 1996. 